\title{
Crescimento somático na população africana em idade escolar. Estado actual do conhecimento
}

\author{
Sílvio Saranga ${ }^{1}$ \\ José Maia ${ }^{2}$ \\ Jorge Rocha ${ }^{3}$ \\ Leonardo Nhantumbo ${ }^{1}$ \\ António Prista ${ }^{1,4}$
}

https://doi.org/10.5628/rpcd.06.01.81

\author{
${ }^{1}$ Universidade Pedagógica, Faculdade de Ciências de \\ Educação Física e Desporto, Maputo, Moçambique \\ ${ }_{2}$ Universidade do Porto, Faculdade de Desporto, Portugal \\ ${ }_{3}^{3}$ Universidade do Porto, Faculdade de Ciências e \\ IPATIMUP, Portugal \\ ${ }^{4}$ Universidade Eduardo Mondlane, Faculdade de Medicina, \\ Maputo, Moçambique
}

\section{RESUMO}

O desenvolvimento da investigação científica em torno do crescimento somático das populações tem-se justificado não só pela sua utilidade no âmbito da saúde pública e desenvolvimento humano, mas também pelo contributo que presta a diferentes domínios da ciência, como sejam a auxologia, a antropologia, a epidemiologia e a nutrição, entre outros. Por se encontrar associado aos estudos das populações afectadas por condições higiénico-nutricionais adversas, o continente africano encontra particular interesse no seu desenvolvimento e aplicação. A presente revisão da literatura, ao tentar estabelecer o estado do conhecimento actual, pretende auxiliar os pesquisadores de populações africanas em idade escolar. A partir da consulta de bases disponíveis, foram seleccionados todos os artigos que versassem estudos realizados em África, com sujeitos em idade escolar e referindo diferentes aspectos do seu crescimento somático. Os estudos revistos revelam uma preocupação centrada em torno do significado antropobiológico do crescimento infanto-juvenil, nomeadamente na influência das condições sócio-económicas no crescimento, sobretudo a partir de um enfoque diferencialista de meios e estratos sócio-económicos distintos. Um outro aspecto de relevo reside no uso generalizado de normas internacionais para avaliar-estimar o estado nutricional, bem como a validade dos pontos de corte usualmente utilizados. Finalmente, sugere-se abordagens mais amplas e diversificadas no entendimento da enorme variabilidade populacional. A epidemiologia genética, ou a genética de populações podem ser enfoques altamente promissores.

Palavras-chave: crescimento, crianças e jovens, África, saúde pública, epidemiologia.

\section{ABSTRACT}

Somatic Growth in African Children and Youth. Current Knowledge

Scientific research in somatic growth has been justified by its utility in public health and human development as well as its major contribution to such fields as auxology, anthropology, epidemiology and nutrition. Since growth deficiencies are associated with adverse hygienic-nutritional conditions, the development and application of knowledge provided from auxology is increasing in Africa. The present literature review has been conducted in order to face the need of a systematisation of the few publications about growth in school aged populations of Africa. All the papers cited in available library databases which refer to studies within this framework of research with school aged African populations have been included. The study of the bio-anthropological meaning of child growth, namely the socio-economic influence, seems to be the main concern. Another issue is the interpretation and validity of using international norms and cut-offs to classify nutritional status. The review also suggests that, facing some of the methodological and substantive limitations of such research and the recent development within the broad field of genetics, new approaches will come out in the near future to solve some of the must relevant research problems.

Key Words: growth, children and youth, Africa, public health, epidemiology. 


\section{INTRODUÇÃO: ÂMBITO, RELEVÂNCIA E ENQUADRAMENTO}

A literatura de cariz antropo-auxológico tem evidenciado, com muita frequência, o papel relevante do estudo do crescimento somático em populações desfavorecidas no âmbito da saúde pública, das avaliações nutricionais e do desenvolvimento pessoal. A infância e a pré-adolescência são considerados períodos sensíveis na vida do ser humano, pelo que a informação circunstanciada e altamente detalhada do crescimento infantil é considerada como um excelente indicador de saúde, ao permitir o estabelecimento de padrões de vigilância do desenvolvimento da criança. Daqui que facilitem o diagnóstico do estado sanitário de populações com propósitos de intervenções adequadas ao nível das comunidades.

Os factores negativos que interfiram precocemente no processo de crescimento, tais como o hipotiroidismo ou a desnutrição, podem ter consequências a longo prazo (22). Uma análise adequada do crescimento permite despistar estes factores, identificar situações em que seja necessária intervenção clínica, bem como avaliar o seu sucesso. Para além deste domínio, em que se pode reduzir substancialmente diferentes aspectos da morbilidade infantil, a prestação de cuidados médicos baseados na monitorização do crescimento tem como objectivo estabelecer as condições ambientais que permitam à criança expressar o seu potencial genético de desenvolvimento. Nos últimos 20 anos, um conjunto variado de estudos centrados em diferentes "lugares" de pesquisa (antropológico, auxológico, epidemiológico, clínico e nutricional) tem apontado, de forma clara, o fosso enorme que separa os resultados de crescimento e desenvolvimento individual e colectivo dos países desenvolvidos, produto de condições sócio-económicas altamente favoráveis, dos que ocorrem nos países em desenvolvimento. Nestes últimos, as crises económicas, de carácter mais ou menos cíclico, conduziram a um agravamento substancial do estado nutricional e da saúde das populações, com fortes prejuízos da sua capacidade de produzir trabalho e fruir de uma vida activa e plena (45). Daqui que não seja surpreendente que o atraso no crescimento somático e desenvolvimento psicomotor das crianças dos países em desenvolvimento, com especial destaque para os da África Sub-Sahariana, seja estabelecido nos primeiros dois ou três anos de vida $(1,19$, 42). Este facto está geralmente associado a uma fraca vigilância pediátrica nestes países devido, entre outros factores, à falta de pessoal técnico e clínico qualificado e a um serviço de saúde deficiente. Olhando para a imensidão territorial e para a grande variabilidade das populações africanas, sobretudo no estrato etário mais jovem, pode afirmar-se, com alguma propriedade, que há relativamente poucos estudos acerca do crescimento e desenvolvimento das populações pertencentes à região Sub-Sahariana, particularmente em subgrupos populacionais rurais. Esta lacuna reclama uma atenção única, se nos concentrarmos na enorme riqueza informacional que estas regiões têm, e que são de grande preciosidade para a interpretação da variabilidade humana e suas implicações nos domínios da medicina, da educação e da qualidade de vida das populações. Devido à sua extraordinária diversidade étnica, geográfica e sóciocultural, a que se associam manifestas assimetrias nos ritmos de desenvolvimento económico, as populações africanas podem ser vistas como experiências naturais em que é possível analisar e ponderar a influência dos diferentes factores que afectam os padrões de crescimento e desenvolvimento humanos. Um olhar pela literatura sobre o crescimento e desenvolvimento em África mostra que há um défice considerável de estudos centrados nas zonas rurais que, juntamente com outras áreas não urbanizadas, concentram mais de $50 \%$ da população africana. $\mathrm{Na}$ sua maioria, os trabalhos de pesquisa têm sido realizados no âmbito da avaliação do estado nutricional, recorrendo a indicadores antropométricos. Por outro lado, as abordagens têm sido essencialmente realizadas com crianças menores de 5 anos, dada a elevada taxa de mortalidade nesta faixa etária. De há alguns anos para cá, começa a notar-se uma tímida, mas emergente, preocupação no estudo da criança em idade escolar, incluindo as diversas fases pubertárias, de que são exemplo os trabalhos realizados por Davies et al. (11), Sellen (42) e Gillett e Tobias (14) na Tanzânia, Cameron et al. (6), Henneberg e Low (18) e Monyeke et al. (28) na África do Sul, Pawloski (32) no Mali, Zverev e Gondwe (54) no Malawi, e, finalmente, em Moçambique por Martins (24), Prista $(33,34,37)$, Muria (29), Saranga (41) e Prista et al. $(35,36,39)$.

Neste trabalho é apresentada uma revisão bibliográfica dos estudos de crescimento realizados em países da África Sub-Sahariana em populações com idade escolar (Quadro 1). 


\begin{tabular}{|c|c|c|c|c|}
\hline Referência e País & Amostra & $\begin{array}{l}\text { Delinea- } \\
\text { mento }\end{array}$ & Variáveis & Principais resultados \\
\hline $\begin{array}{l}\text { Martins [1971]. Height, } \\
\text { Weight and Chest } \\
\text { Circumference of children of } \\
\text { different ethnic groups in } \\
\text { Lourenço Marques, } \\
\text { Mozambique, in } 1965 \text { with a } \\
\text { note on the secular trend. } \\
\text { Moçambique }\end{array}$ & $\begin{array}{l}14640 \text { crianças e jovens } \\
\text { com idades } \\
\text { compreendidas entre os } \\
6-18 \text { anos. }\end{array}$ & 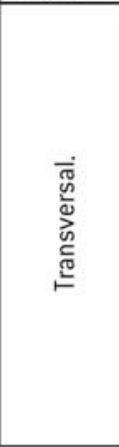 & $\begin{array}{l}\text { Altura, peso, } \\
\text { circunferência } \\
\text { do tórax. }\end{array}$ & $\begin{array}{l}\text { Os rapazes tinham maior estatura e } \\
\text { peso em todas as idades, enquanto que } \\
\text { as raparigas, só até aos } 14 \text { anos de } \\
\text { idade. Dados disponíveis de crianças } \\
\text { caucasianas de há } 35 \text { anos atrás } \\
\text { mostram uma tendência secular para } \\
\text { altura de } 0.5 \mathrm{~cm} \text { aos } ? \text { anos nos } \\
\text { rapazes, e } 1.5 \mathrm{~cm} \text {, nas raparigas, } \\
\text { encontrando-se muito abaixo da } \\
\text { tendência ocorrida com populações } \\
\text { europeias da mesma idade. }\end{array}$ \\
\hline $\begin{array}{l}\text { Davies et al. (1974). } \\
\text { Physical growth and } \\
\text { development of urban and } \\
\text { rural East African children. } \\
\text { Tanzania. }\end{array}$ & $\begin{array}{l}654 \text { sujeitos urbanos } \\
\text { ( } 340 \text { rapazes e } 314 \\
\text { raparigas) e } 384 \\
\text { indivíduos rurais [ } 248 \\
\text { rapazes e } 136 \text { raparigas] } \\
\text { com idades } \\
\text { compreendidas entre os ?. } \\
16 \text { anos. }\end{array}$ & 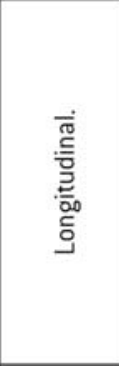 & $\begin{array}{l}\text { Altura, peso, } \\
\text { pregas de } \\
\text { adiposidade e } \\
\text { circunferência } \\
\text { de braço. }\end{array}$ & $\begin{array}{l}\text { Não foram observadas diferenças } \\
\text { significativas entre os dois grupos na } \\
\text { altura, peso, pregas de adiposidade e } \\
\text { circunferência de braço. É sugerido que } \\
\text { as crianças da África Austral são baixas } \\
\text { e magras em relação a crianças } \\
\text { europeias, mas a idade em que é } \\
\text { atingido o pico de velocidade em altura e } \\
\text { peso é aproximadamente a mesma. }\end{array}$ \\
\hline $\begin{array}{l}\text { Prista et al, (1997). } \\
\text { Relationship between } \\
\text { physical activity, } \\
\text { socioeconomic status and } \\
\text { physical fitness of } 8-15 \\
\text { year old youth from } \\
\text { Mozambique. Moçambique }\end{array}$ & $\begin{array}{l}593 \text { crianças [ } 277 \\
\text { rapazes e } 316 \text { raparigas] } \\
\text { com idades entre os } 8-15 \\
\text { anos. }\end{array}$ & 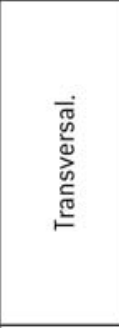 & $\begin{array}{l}\text { Altura, peso, } \\
\text { pregas de } \\
\text { adiposidade. }\end{array}$ & $\begin{array}{l}\text { Valores de estatura inferiores às normas } \\
\text { internacionais, reflectindo um atraso } \\
\text { maturacional. Influência significativa do } \\
\text { estatuto socioeconómico no } \\
\text { crescimento. Os valores de peso e de } \\
\text { massa muscular analisados em função } \\
\text { da altura eram próximos dos valores } \\
\text { considerados normais. }\end{array}$ \\
\hline $\begin{array}{l}\text { Prista (1998). } \\
\text { Anthropometric indicators } \\
\text { of nutritional status: } \\
\text { Implications for fitness, } \\
\text { activity, and health in } \\
\text { school-age children and } \\
\text { adolescents from Maputo. } \\
\text { Moçambique }\end{array}$ & $\begin{array}{l}593 \text { crianças [ } 277 \\
\text { rapazes e } 316 \text { raparigas] } \\
\text { com idades entre os } 8-15 \\
\text { anos. }\end{array}$ & 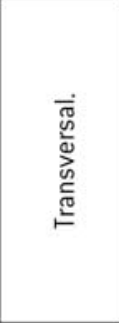 & $\begin{array}{l}\text { Altura, peso, } \\
\text { gordura } \\
\text { corporal, } \\
\text { aptidão física, } \\
\text { aptidão } \\
\text { metabólica, } \\
\text { actividade } \\
\text { física. }\end{array}$ & $\begin{array}{l}\text { Não foi encontrada relevância nos } \\
\text { pontos de corte adoptados pela OMS } \\
\text { para classificar subnutrição. }\end{array}$ \\
\hline
\end{tabular}




\begin{tabular}{|c|c|c|c|c|}
\hline $\begin{array}{l}\text { Muria (1998). Efeito das } \\
\text { condições sócio- } \\
\text { económicas, maturação e } \\
\text { do crescimento somático na } \\
\text { aptidão física de crianças e } \\
\text { jovens da cidade de Maputo } \\
\text { de ambos os sexos com } \\
\text { idades compreendidas } \\
\text { entre os } 8 \text { e os } 11 \text { anos. } \\
\text { Moçambique }\end{array}$ & $\begin{array}{l}547 \text { crianças [ } 258 \\
\text { rapazes e } 289 \text { raparigas] } \\
\text { com idades entre os } 8-11 \\
\text { anos. }\end{array}$ & 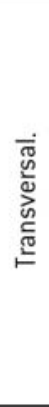 & $\begin{array}{l}\text { Altura, peso, } \\
\text { pregas } \\
\text { tricipital e } \\
\text { subescapular. }\end{array}$ & $\begin{array}{l}\text { Foram encontrados padrões de altura e } \\
\text { peso relativos ao dimorfismo sexual } \\
\text { idênticos aos descritos na literatura } \\
\text { para outras populações. Influência } \\
\text { significativa do estatuto } \\
\text { socioeconómico nas dimensões } \\
\text { corporais. }\end{array}$ \\
\hline $\begin{array}{l}\text { Henneberg e Louw (1998]. } \\
\text { Cross-sectional Survey of } \\
\text { growth of urban and rural } \\
\text { "Cape Coloured" } \\
\text { schoolchildren: } \\
\text { anthropometry and } \\
\text { functional tests. África do } \\
\text { Sul. }\end{array}$ & $\begin{array}{l}1974 \text { individuos ( } 906 \\
\text { rapazes e } 1068 \text { raparigas] } \\
\text { de estatuto sócio- } \\
\text { económico elevado, } \\
\text { idades entre os } 5 \text { e } 20 \\
\text { anos e } 1774 \text { indivíduos } \\
\text { (834 rapazes e } 940 \\
\text { raparigas) de estatuto } \\
\text { socioeconómico baixo, } \\
\text { idades entre } 5 \text { a } 19 \text { anos. }\end{array}$ & 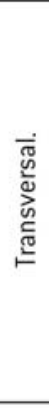 & $\begin{array}{l}\text { Altura, peso, } \\
\text { prega tricipital } \\
\text { e subescapular }\end{array}$ & $\begin{array}{l}\text { Os valores de altura e peso das crianças } \\
\text { do meio rural abaixo dos valores das } \\
\text { crianças do meio urbano. No que se } \\
\text { refere à composição corporal, as } \\
\text { crianças urbanas encontram-se acima } \\
\text { das normas de referência americanas. }\end{array}$ \\
\hline $\begin{array}{l}\text { Sellen (1999). Growth } \\
\text { patterns among semi } \\
\text { nomadic pastoralists } \\
\text { (Datoga) of Tanzânia. } \\
\text { Tanzânia. }\end{array}$ & $\begin{array}{l}470 \text { crianças semi- } \\
\text { nómadas, idades entre os } \\
0.18 \text { anos. }\end{array}$ & 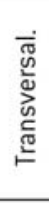 & $\begin{array}{l}\text { Altura, peso, } \\
\text { prega tricipital, } \\
\text { subescapulare } \\
\text { circunferência } \\
\text { do braço. }\end{array}$ & $\begin{array}{l}\text { Foi possível observar um défice de } \\
\text { crescimento estabelecido nos primeiros } \\
2.3 \text { anos de vida. Quando a comparação } \\
\text { é feita com populações de referência o } \\
\text { défice torna-se ainda maior. }\end{array}$ \\
\hline $\begin{array}{l}\text { Zverev e Gondwe [2001]. } \\
\text { Growth of urban school } \\
\text { children in Malawi. } \\
\text { Malawi. }\end{array}$ & $\begin{array}{l}493 \text { crianças com idades } \\
\text { entre os } 6-17 \text { anos. }\end{array}$ & 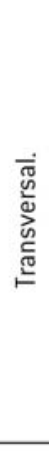 & $\begin{array}{l}\text { Altura, peso, } \\
\text { circunferência } \\
\text { de braço, prega } \\
\text { trícipital, } \\
\text { circunferência } \\
\text { do braço. }\end{array}$ & $\begin{array}{l}\text { Valores inferiores aos valores de } \\
\text { referência da OMS. Crianças mais velhas } \\
\text { de ambos os sexos tiveram valores } \\
\text { elevados na prega tricipital quando } \\
\text { sobrepostos aos valores de referência } \\
\text { internacionais, enquanto que nas } \\
\text { crianças mais novas o fenómeno foi } \\
\text { inverso. Os adolescentes aproximam-se } \\
\text { dos valores de referência da OMS e o } \\
\text { padrão encontrado assemelha-se ao de } \\
\text { outras populações da região. }\end{array}$ \\
\hline $\begin{array}{l}\text { Monyeki et al. (2000). } \\
\text { Growth and nutritional } \\
\text { status of rural South African } \\
\text { children 3-10 years old: The } \\
\text { Ellisras growth study. África } \\
\text { do Sul. }\end{array}$ & $\begin{array}{l}1335 \text { crianças [ } 684 \\
\text { rapazes, } 651 \text { raparigas] } \\
\text { com idades } \\
\text { compreendidas entre os } 3 \text { - } \\
10 \text { anos. }\end{array}$ & 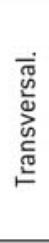 & $\begin{array}{l}\text { Altura, peso, } \\
\text { IMC }\end{array}$ & $\begin{array}{l}\text { Encontrada uma elevada prevalência de } \\
\text { stunting, sendo de } 10 \% \text { aos } ? \text { anos e } \\
\text { mais de } 30 \% \text {, aos } 10 \text {. Atraso em relação } \\
\text { às normas da OMS. }\end{array}$ \\
\hline
\end{tabular}




\begin{tabular}{|c|c|c|c|c|}
\hline $\begin{array}{l}\text { Sallen (2000). Seasonal } \\
\text { ecology and nutritional } \\
\text { status of women and } \\
\text { children in Tanzanian } \\
\text { pastoral community. } \\
\text { Tanzania. }\end{array}$ & $\begin{array}{l}180 \text { mulheres e } 347 \\
\text { crianças e jovens com } \\
\text { idades entre os } 0-18 \text { anos. }\end{array}$ & 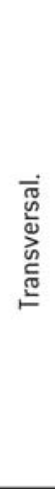 & Altura e peso. & $\begin{array}{l}\text { Elevada prevalência de desnutrição } \\
\text { entre mulheres e crianças com } \\
\text { IMC<18.5. Diminuições sazonais } \\
\text { moderadas dos niveis de gordura } \\
\text { corporal foram observadas. Mais de } \\
\text { metade das crianças mostram um } \\
\text { evidente atraso no crescimento } \\
\text { somático devido a desnutrição. A } \\
\text { redução da gordura corporal em } \\
\text { crianças é evidente na estação seca } \\
\text { relativamente à estação chuvosa, } \\
\text { embora de magnitude reduzida. }\end{array}$ \\
\hline $\begin{array}{l}\text { Saranga [2001]. Variação de } \\
\text { curta duração no } \\
\text { crescimento somático e } \\
\text { aptidão física de crianças e } \\
\text { jovens da cidade de Maputo, } \\
\text { Moçambique. Influência das } \\
\text { alterações sócio- } \\
\text { económicas. Moçambique }\end{array}$ & $\begin{array}{l}593 \text { crianças avaliadas } \\
\text { em } 1992 \text { [ } 276 \text { rapazes e } \\
317 \text { raparigas) e } 2156 \\
\text { crianças em estudo de } \\
1999 \text { (1016 rapazes e } \\
1140 \text { raparigas) com } \\
\text { idades entre os } 8-15 \text { anos. }\end{array}$ & 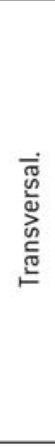 & $\begin{array}{l}\text { Altura, peso, } \\
\text { pregas } \\
\text { tricipitale } \\
\text { subescapular. }\end{array}$ & $\begin{array}{l}\text { As crianças estudadas em } 1999 \\
\text { apresentam maior estatura, peso e } \\
\text { percentagem de gordura corporal } \\
\text { quando comparadas com as do estudo } \\
\text { de } 1992.0 \text { maior peso das crianças } \\
\text { estudadas em } 1999 \text { deve-se a } \\
\text { incrementos substanciais de gordura } \\
\text { corporal. Conclui-se que as alterações } \\
\text { estavam associadas a abruptas } \\
\text { modificações sócio-politicas e } \\
\text { económicas registadas naquele período. }\end{array}$ \\
\hline $\begin{array}{l}\text { Gillett e Tobias [2002]. } \\
\text { Human growth in Southern } \\
\text { Zâmbia: A First Study of } \\
\text { Tonga Children Predating } \\
\text { the Kariba Dam [1957. } \\
\text { 1958). Tanzânia. }\end{array}$ & $\begin{array}{l}602 \text { crianças [ } 386 \\
\text { rapazes e } 216 \text { raparigas ] } \\
\text { idades entre os } 6-13 \text { anos }\end{array}$ & 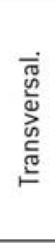 & Altura e peso. & $\begin{array}{l}\text { Diferenças significativas entre as } \\
\text { crianças de duas áreas distintas, que } \\
\text { parecem ser explicadas por factores } \\
\text { nutricionais. }\end{array}$ \\
\hline $\begin{array}{l}\text { Pawloski (2003). Mixed- } \\
\text { longitudinal analysis of } \\
\text { growth data from Malian } \\
\text { adolescent girls: Evidence } \\
\text { for compensatory gain? } \\
\text { Mali. }\end{array}$ & $\begin{array}{l}1045 \text { raparigas } \\
\text { adolescentes com idades } \\
\text { compreendidas entre os } \\
10-17 \text { anos. }\end{array}$ & 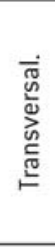 & Altura, peso. & $\begin{array}{l}\text { Estatura e peso inferiores às normas de } \\
\text { referência internacionais. }\end{array}$ \\
\hline $\begin{array}{l}\text { Prista et al. [2003]. } \\
\text { Anthropometric indicators } \\
\text { of nutritional status } \\
\text { implications for fitness, } \\
\text { activity, and health in } \\
\text { school-age children and } \\
\text { adolescents from Maputo, } \\
\text { Mozambique. } \\
\text { Moçambique }\end{array}$ & $\begin{array}{l}2316 \text { crianças [ } 1094 \\
\text { rapazes e } 1222 \text { raparigas] } \\
\text { com idades entre os } 6-18 \\
\text { anos. }\end{array}$ & 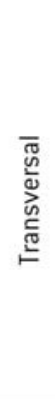 & $\begin{array}{l}\text { Estado clínico, } \\
\text { aptidão } \\
\text { metabólica, } \\
\text { parasitologia, } \\
\text { altura, peso, } \\
\text { gordura } \\
\text { corporal, } \\
\text { aptidão física, } \\
\text { actividade } \\
\text { física. }\end{array}$ & $\begin{array}{l}\text { Não foi encontrada relevância nos } \\
\text { critérios para classificar subnutridos na } \\
\text { população estudada. Os valores de corte } \\
\text { para definir sobrepeso e obesidade } \\
\text { mostraram-se válidos. }\end{array}$ \\
\hline
\end{tabular}


A fim de sistematizar os principais tópicos abordados nestas pesquisas, a apresentação será dividida em duas áreas temáticas fundamentais: 1) significado regional e nacional dos padrões de crescimento e desenvolvimento, suas associações com indicadores de saúde e comparações com dados de referência internacional; 2) comparações entre os padrões observados em populações rurais e urbanas e em diferentes grupos sócio-económicos.

É objectivo do presente documento a descrição dos trabalhos publicados sobre estudos com populações locais africanas, a sistematização dos principais problemas e resultados, bem como a tentativa de perspectivar os eixos principais de desenvolvimento da investigação futura.

\section{VARIAÇÃO REGIONAL DOS PADRÕES DE CRESCIMENTO E SUAS RELAÇÕES COM DADOS DE REFERÊNCIA INTERNACIONAIS}

A altura e o peso são dois dos indicadores antropométricos de maior relevância na elucidação do estado nutricional, assumindo-se, geralmente, que desvios negativos do desenvolvimento estaturo-ponderal de uma criança traduzem deficiência nutricional (33, 34, 36). Estes desvios, denominados de stunting (baixa estatura em relação à idade, ou atraso na velocidade de crescimento linear) e wasting (baixo peso em relação à estatura ou atraso na velocidade de crescimento ponderal), estão bem documentados em países em desenvolvimento $(25,43,52)$. A qualidade de um critério de diagnóstico baseado na definição de um limite de normalidade pode ser avaliada pela sua sensibilidade e especificidade. A sensibilidade é uma medida do poder de identificação de casos patológicos. Será tanto mais alta quanto menor a percentagem de falsos negativos. A especificidade quantifica a fiabilidade na identificação de casos normais e será tanto mais alta quanto menor a percentagem de falsos positivos. Se, por exemplo, procurarmos aumentar a sensibilidade através de uma deslocação do limite de normalidade para o percentil 10, haverá uma redução da especificidade. Se o limite de normalidade for menor que o percentil 3 , verificarse-á o inverso. Normalmente, entende-se por desvio da taxa normal a perda da posição percentílica com trajectórias para canais percentílicos abaixo do per- centil 5. O limite inferior do canal geralmente admitido no âmbito da pediatria clínica é o percentil 3. A Organização Mundial de Saúde recomenda o uso de normas dos Centros Nacionais de Estatísticas de Saúde, com destaque para as oriundas de estudos realizados com crianças americanas (49), quando se pretende obter uma base de contraste em estudos comparativos independentes de populações e de subgrupos populacionais (49). Contudo, esta sugestão e prática têm sido objecto de várias críticas $(36,39)$. Um dos principais constrangimentos nas práticas pediátricas é a ambiguidade na definição dos parâmetros do crescimento normal. A ausência de uma definição precisa e inequívoca de stunting e wasting impede a identificação clara das crianças em risco. A incapacidade para detectar estes fenómenos tem fortes implicações no diagnóstico de certas doenças, visto que estes desvios são, na sua essência, diferentes e têm significados clínicos distintos. Este problema tem sido objecto de inúmeros debates, sobretudo no que respeita à utilidade relativa do uso de normas internacionais ou nacionais. Actualmente parece haver um consenso, mais ou menos generalizado, relativamente à vantagem da utilização de normas nacionais, isto é, normas provenientes de amostras da população em análise, constituídas por indivíduos com estado de saúde óptimo e não expostas a constrangimentos sérios ou agressões ambientais. No entanto, a ausência deste tipo de normas na maior parte dos países tem levado a uma maior utilização dos padrões de referência internacionais actualmente disponíveis. Segundo Tanner (44), as normas internacionais são válidas para crianças de diversos grupos sócio-económicos, pelo que as estaturas e pesos que se encontrem dentro dos canais percentílicos das tabelas de referência podem ser consideradas normais.

A grande controvérsia, no que se refere a comparações inter-populacionais usando estas referências, resulta, por um lado, do facto de haver uma grande variabilidade étnica nas populações e, por outro, dos grupos de referência serem geralmente de origem europeia e de classes sociais e ambientes específicos. Embora seja consensual que as normas de referência devam ser determinadas a partir de populações saudavelmente nutridas e apresentando estados clínicos 
normalizados, considera-se que os desvios destas normas podem não indicar necessariamente situações patológicas. Assim, salientando a importância da variabilidade étnico-regional como fruto de uma interacção genética e ambiental natural, a União Internacional de Ciências Nutricionais propunha, em 1971, que o uso de valores de referência locais permitiria uma mais fácil identificação da posição do sujeito, tendo em conta as condições específicas do seu ambiente, incluindo a nutrição e os serviços de saúde $(15,17,23,30,46)$. Neste contexto, as tabelas padronizadas a partir de outras populações devem ser, no entender de alguns autores, designadas como referências e não como normas $(50,7)$. A adopção de normas locais apresenta, contudo, constrangimentos importantes. Em primeiro lugar, o facto da sua construção ser muito onerosa, exigir grandes amostras e a formação de técnicos locais com elevados níveis de proficiência tornar a sua execução muito difícil. Em segundo lugar, a elevada prevalência das mais variadas doenças e sequelas de condições higiénico-nutricionais adversas dificulta a definição de critérios seguros de inclusão e exclusão. Face a estas dificuldades de construção de normas locais, tem sido considerado que as normas de referência universais, apesar das suas limitações, constituem o meio disponível mais apropriado para o despiste dos atrasos nos índices de crescimento das populações (16). Esta opção é apoiada pela observação de que as crianças dos grupos sócio-económicos mais prósperos de diferentes populações apresentam menor disparidade de crescimento linear do que subgrupos da mesma população pertencentes a diferentes estratos sócio-económicos (17). Tal padrão parece indicar que as diferenças ambientais intrapopulacionais se sobrepõem às possíveis diferenças genéticas inter-populacionais (26).

Pelo contrário, outros autores sustentam que a diferenciação genética pode ter implicações significativas nos padrões de crescimento dos diversos grupos étnicos, explicando, por exemplo, as diferenças observadas entre europeus e asiáticos $(15,40,12)$. No entender destes autores, o uso de normas de referência exteriores à população em estudo pode conduzir a interpretações enviesadas das implicações dos resultados, devido à falta de indicadores sensíveis e fiáveis do atraso no crescimento, como a morbilidade e mortalidade $(1,19)$. A importância destes indicadores reside no facto de poderem servir de caracterizadores da população, para além de facilitarem a comparação com populações de países desenvolvidos. Para as populações africanas ainda não há ferramentas baseadas em evidências empíricas para medir a morbilidade, devido, entre outras coisas, a carências estruturais e formais dos serviços de saúde.

Dois trabalhos recentes nesta matéria foram realizados em Moçambique $(36,39)$. Em dois momentos diferentes, os autores avaliaram a relevância biológica dos critérios antropométricos de avaliação do estado nutricional adoptados pela OMS (52) em populações de idade escolar de ambos os sexos. A pesquisa consistiu, essencialmente, na divisão de duas grandes amostras em grupos nutricionais de acordo com a classificação da OMS, e na comparação das mesmas em termos clínicos, de performance motora e actividade física habitual, assumindo que um estado nutricional deficiente se reflectiria necessariamente, e negativamente, nas variáveis em estudo. Desta investigação resultou a conclusão que os valores de corte para definir sujeitos com subnutrição (wasted e stunted) não apresentavam validade, dado que as poucas diferenças encontradas eram explicadas apenas pelas diferenças em tamanho corporal, o que conduziu à discussão acerca dos efeitos e significado alométrico da estatura. Por outro lado, foi sugerida validade para os critérios de definição de obesidade, já que se observaram diferenças significativas entre os classificados de obesos e os restantes grupos nutricionais. Apesar de pesquisar populações de idade pré-escolar, van Loon (46) demonstrou que o uso de critérios desajustados poderá induzir erros com implicações humanas de impacto negativo. Por exemplo, a sobre-classificação de prevalência de malnutrição pode levar ao abandono de estratégias eficientes, por causa das estimativas de custos operativos que poderiam ser realizados se o número de falsos positivos não fosse tão elevado. Parece ser de concluir, daqui, a urgência do desenvolvimento e afinamento de instrumentos simples e altamente fiáveis, de validade biológica comprovada para classificar, com elevado rigor e segurança, diferentes estados nutricionais. 


\section{COMPARAÇÕES ENTRE OS PADRÕES OBSERVADOS EM POPULAÇÕES RURAIS E URBANAS E EM DIFERENTES GRUPOS SÓCIO-ECONÓMICOS.}

Cameron $(4,5)$, baseando-se em dados longitudinais de crianças sul-africanas de áreas rurais, demonstrou que os seus padrões de crescimento se encontravam muito abaixo do percentil 50 até ao período da adolescência. No entanto, após o salto pubertário, os valores atingidos não se encontravam muito longe dos padrões dos jovens americanos e britânicos. O mesmo autor (5), num estudo de revisão, comparou os valores de crescimento (altura e peso) e constatou que havia diferenças significativas entre raparigas sul-africanas de áreas urbanas e rurais. Refere que crianças de nível sócio-económico elevado se encontram muito próximo do percentil 50 das normas da OMS. Por outro lado, observou que crianças rurais e urbanas de nível sócio-económico elevado demonstram melhores índices de crescimento do que crianças urbanas de nível socioeconómico mais baixo. Numa pesquisa posterior, Cameron (4) observou uma menor velocidade de crescimento, seguida de um longo período de crescimento que vai até aproximadamente aos vinte anos.

Para autores como Eveleth e Tanner (13), Bogin (3) e Meredith (27), crianças de estatuto socioeconómico elevado que vivem em meio urbano são, de forma geral, mais altas, mais pesadas e com uma maior percentagem de gordura corporal, devido ao facto do meio em que vivem estar muitas vezes associado a um estatuto socioeconómico elevado. Contudo, é de referir que crianças urbanas de estatuto socioeconómico médio são, com algumas raras excepções, baixas e têm um estatuto nutricional inferior ao das crianças de populações rurais.

Pawloski (31) investigou o crescimento de raparigas rurais e urbanas do Mali. Observou um atraso estaturo-ponderal quando comparou os seus valores com raparigas americanas de referência. No que se refere ao índice de massa corporal, os resultados não revelaram a presença de desnutrição proteica. Raparigas malianas de áreas rurais, contrastadas com as de áreas urbanas, apresentam baixo peso e menor estatura e, consequentemente, um índice de massa corporal inferior. Henneberg e Louw (18) realizaram um estudo denominado Cross-sectional survey of growth of urban and rural cape coloured schoolchildren: anthropometry and functional tests, envolvendo dois grupos de crianças urbanas e rurais, com o máximo de contraste no que se refere ao estatuto sócio-económico na África do Sul. Os dados foram recolhidos entre os anos 1986 e 1988, numa amostra de 1974 crianças urbanas do estatuto sócioeconómico elevado (906 rapazes e 1068 raparigas), com idades compreendidas entre os 5 e 20 anos; numa outra amostra de 1774 crianças rurais de estatuto socioeconómico baixo (834 rapazes e 940 raparigas), as idades estavam compreendidas entre os 5 e 19 anos. Tomaram como indicadores do padrão de crescimento a altura, o peso, o comprimento dos membros, perímetros do braço e da coxa. Verificou-se que, em média, as crianças do meio rural se encontram abaixo das crianças do meio urbano nos indicadores altura e peso. Quanto à composição corporal, as crianças urbanas encontram-se acima das normas de referência americana. Pawloski (32), a partir de uma pesquisa efectuada numa amostra de 1045 raparigas do Mali com idades compreendidas entre os 10-17 anos, levantou as seguintes hipóteses de trabalho: 1) as raparigas exibem menores valores de crescimento, quando comparadas com outras bem nutridas; 2) as raparigas de áreas rurais exibem baixos valores estaturais, quando comparadas com raparigas malianas de áreas urbanas; 3 ) as raparigas que não frequentam a escola são também mais baixas do que as que frequentam a escola; e 4) ganhos compensatórios são evidentes nas adolescentes. Os resultados revelaram um fraco crescimento e desenvolvimento em adolescentes do sexo feminino no Mali e uma menor estatura e peso, quando comparadas com raparigas da população americana. Estudos realizados por autores como Wagstaff et al. (48), Chaning-Pearce e Solomon (8) sugerem que o evidente atraso no crescimento e desenvolvimento é devido a uma combinação de factores, dos quais se destacam: os alimentos com baixo valor nutritivo, o alto dispêndio energético nas actividades diárias e a fraca qualidade dos cuidados primários de saúde. As adolescentes seguem raramente um padrão standard de crescimento, devido a diferentes timings biológicos e à expressão diferenciada do crescimento na puberdade.

Recentemente, Monyeki et al. (28) conduziram um estudo transversal com o objectivo de conhecer o estado nutricional e de crescimento de crianças sul 
africanas usando como indicadores as rácios de altura-pela-idade, peso-pela-idade e índice de massa corporal de crianças de 3 a 10 anos de idade. A amostra, de uma aldeia rural a norte da África do Sul, foi constituída por 1335 crianças (684 rapazes, 651 raparigas). Os resultados sugerem uma alta prevalência de stunting, que vai dos $10 \%$, aos 7 anos, para mais de $30 \%$, aos 10 anos de idade. A sobreposição dos resultados deste estudo às normas de referência identificou um atraso nos níveis de crescimento das crianças desta amostra, provavelmente devido às condições stressantes em que vivem. No entanto, é de referir que, se a condição de stunting durante a infância for permanente, pode conduzir a uma perda de capacidade para produzir trabalho quando estes indivíduos forem adultos. Investigações realizadas por Wagstaff et al. (48) e Chaning-Pearce e Solomon (8) monstraram que, em média, crianças negras do bairro do Soweto, arredores de Joanesburgo, tinham valores mais baixos na estatura e peso, quando comparadas com os seus pares de origem europeia ou com as normas americanas usadas como referência pela OMS, apesar de aparentemente gozarem dos aspectos positivos da vida urbana, como provisão nutricional e fácil acesso aos cuidados primários de saúde.

Do ponto de vista social, o estatuto socioeconómico, a dimensão das famílias e o envolvimento social parecem ser causas que interferem no processo de crescimento, para além das componentes étnica ou racial e climática. Num estudo transversal com populações de países em desenvolvimento, Victora (47) observou que os resultados das rácios antropométricas diferiam em várias regiões do planeta. Constatou, por exemplo, que crianças e jovens de países da América Latina apresentavam prevalências muito elevadas de stunting e baixas no que se refere a wasting, enquanto que nos países asiáticos havia uma alta prevalência, tanto de stunding como de wasting. O mesmo autor não atribui um papel de relevo aos factores genéticos, referindo que estes padrões de resultados podiam ser atribuídos a diferenças nas condições socioeconómicas daqueles países.

O mais provável é que todas as crianças dos diversos grupos étnicos apresentem um potencial genético para o crescimento linear bastante similar quando as condições ambientais são óptimas. A constatação da variabilidade étnica no crescimento encontra suporte em estudos realizados por autores como Habicht et al. (17), Graitcer e Gentry (16), Martorell (25), OMS (53), Keller (20), Kow et al. (21). Na perspectiva de Beaton et al. (1) as diferenças socioeconómicas intra-populacionais estão na origem das diferenças na velocidade de crescimento estatural, devido principalmente a circunstâncias ambientais e não a diferenças étnicas no potencial de crescimento, pelo menos até aos cinco anos.

Na Tanzânia, Davies et al. (11) realizaram um estudo longitudinal denominado Physical growth and development of urban and rural East African children.

Acompanharam, durante sete anos, uma amostra de 340 rapazes e 314 raparigas de áreas urbanas e 248 rapazes e 136 raparigas de áreas rurais. As idades estudadas estavam compreendidas entre os 7-16 anos. Pretendiam analisar o crescimento estatural, o peso e a composição corporal de crianças Bantu da zona austral de África. Não foi encontrada qualquer diferença significativa entre os dois grupos, quer no sexo masculino quer no feminino, no que se refere aos padrões da estatura, peso, pregas de adiposidade subcutânea e perímetro braquial. Quando sobrepuseram os valores destas crianças e jovens aos das normas da OMS, os resultados mostraram que as crianças da África Austral eram baixas e magras em relação a crianças europeias, mas o momento em que ocorre a idade do pico de velocidade em altura e peso é aproximadamente o mesmo.

Corlett (9), num projecto denominado Growth of urban school children in Botswana estudou um total de 721 crianças com idades compreendidas entre os $6 \mathrm{e}$ os 15 anos, pretendendo descrever o estatuto de crescimento das crianças de Gaborone, capital do Botswana. Dos resultados, pode-se concluir que rapazes e raparigas, em todas as idades, têm altura e peso abaixo do percentil 50 das normas de referência britânicas e americanas, e abaixo do percentil $10 \mathrm{da}$ OMS. No que se refere aos valores do peso em função da estatura, constatou-se uma frequência reduzida de crianças nos percentis mais baixos da distribuição destas variáveis somáticas. Neste mesmo indicador, é notório um padrão de desenvolvimento semelhante ao registado em outros estudos com populações africanas. No que se refere ao perímetro braquial, ao sobreporem os resultados obtidos nas normas britânicas, as diferenças observadas ficaram 
a dever-se à menor estatura e peso das crianças tswanas e a diferenças no tempo de crescimento. Diferenças semelhantes são também observadas entre crianças tswanas e outros subgrupos populacionais africanos.

Embora o primeiro trabalho conhecido seja do ano de 1968 (24), tendo como indicadores a estatura e o peso das crianças das escolas da cidade de Lourenço Marques (actual Maputo), é nos últimos quinze anos que emerge de modo substancial e continuado uma vaga de publicações sobre a populações moçambicanas de idade escolar $(29,34,35,37,39,41)$.

O estudo de Martins (24) conclui a existência de uma associação da taxa de crescimento às condições sócio-económicas, muitas vezes confundidas com o factor raça. Depois de um interregno de 26 anos, durante o qual nada foi publicado de importante neste domínio, foi realizado na cidade de Maputo um trabalho integrado num estudo sobre a Influência da actividade física e dos factores sócio-económicos sobre as componentes da estrutura do valor físico relacionado a saúde (33). Com uma amostra de 593 indivíduos (277 rapazes e 316 raparigas), de idades compreendidas entre os 8 e 15 anos, foi possível constatar que os indicadores somáticos da população de Maputo eram inferiores às normas internacionais, reflectindo, provavelmente, um atraso maturacional provocado por condições higiénico-nutricionais adversas. Este projecto de pesquisa trouxe, comparativamente ao de 1968, um conjunto de informações adicionais, devido ao facto de a comparação entre estatutos socioeconómicos, vulgo "ricos" e "pobres", se ter realizado entre crianças e jovens da mesma origem étnica, separados por condições de vida diferentes e apenas por uma geração. Verificou-se uma elevada prevalência de stunting e wasting e questionou-se a validade dos critérios antropométricos adoptados para avaliar o estado nutricional conducente a esta situação, e que já foram anteriormente referenciados. Esta investigação gerou novas hipóteses de trabalho e inventariou um novo conjunto de problemas que, desde então, têm vindo a ser continuamente realizados através de vários estudos centrados em diferentes regiões da cidade de Maputo. Na generalidade têm evidenciado uma grande associação entre as condições sócioambientais, crescimento somático, maturação biológica e composição corporal $(29,41)$.
Utilizando uma amostra de 2156 crianças e jovens dos 8 aos 18 anos de idade (1016 rapazes, 1140 raparigas), Saranga et al. (41), avaliaram a variação no crescimento somático das crianças e jovens da cidade de Maputo entre os anos de 1992 e 1999. Tendo como objectivo verificar em que medida as grandes alterações das condições sócio-ambientais verificadas em Maputo naquele período de tempo, resultantes da passagem de uma situação de guerra generalizada para uma situação de paz e crescimento económico, se repercutiam no crescimento somático, os autores salientaram que: 1) as crianças estudadas em 1999, depois da guerra, apresentam maior estatura, peso e percentagem de gordura corporal, quando comparadas com as do estudo de 1994; 2) o aumento do peso das crianças estudadas em 1999 deveu-se a um incremento substancial da gordura corporal, e que 3) as diferenças socioeconómicas, quando expressas em função do indicador altura, se agudizavam (41).

\section{CONCLUSÕES}

Em síntese, apesar da relativa escassez de estudos de crescimento e desenvolvimento em populações africanas, a pesquisa disponível sugere claramente que, embora as trajectórias de crescimento na maioria das amostras estudadas sejam semelhantes às encontradas nas normas internacionais, as médias de altura são consistentemente mais baixas, situando-se no percentil 5 aos cinco anos, decrescendo até a adolescência, devido a uma diminuição da velocidade de crescimento, e seguindo, posteriormente, uma recanalização. Os ganhos compensatórios durante o salto pubertário servem para recuperar perdas de crescimento da infância. No entanto, o grau de reposição das insuficiências de velocidades de crescimento estaturo-ponderais da infância ainda não é totalmente conhecido.

Os trabalhos mostram uma alta prevalência de stunting e atraso no crescimento de crianças rurais na África Sub-Sahariana em relação aos seus pares das áreas urbanas. Porém, nem sempre o ambiente urbano resulta numa melhoria de crescimento estatural. As elevadas taxas de urbanização nestes países, com o consequente aumento de áreas urbanas, resultam em grandes "comunidades urbanas informais" nas quais, por vezes, o estado de crescimento é inferior ao das crianças das comunidades rurais. 
Embora nos estudos consultados tenha havido a preocupação de desenvolver padrões locais de referência para o peso, altura e índices antropométricos, emerge a necessidade de resolver a polémica que advém do uso de normas de referência internacional na estimativa e predição de malnutrição em crianças dos países africanos.

Não obstante a importância da pesquisa realizada, bem como a riqueza e extensão da sua informação, a investigação no domínio do crescimento em África carece de continuidade, na medida em que, para além da avaliação da consistência dos resultados, os avanços científicos permitirão ampliar a abrangência dos estudos em domínios até aqui não explorados. Por exemplo, há a considerar que a maior parte dos trabalhos se resume a regiões extremamente demarcadas, não se podendo, por isso, generalizar os seus resultados face a uma população supostamente muito diversificada em termos ambientais, genéticos, étnicos e sócio-económicos. Neste contexto, é importante que futuros estudos possam vir a preencher estas lacunas, nomeadamente através da extensão da cobertura territorial respeitando a orografia, e abrindo-se a incursões na epidemiologia genética e do ensaio da construção de normas do padrão de crescimento.

\section{CORRESPONDÊNCIA}

Sílvio Pedro José Saranga

Universidade Pedagógica

Faculdade de Ciências de Educação Física e Desporto

Caixa Postal 2107

Maputo, Moçambique

silviosaranga@hotmail.com 


\section{BIBLIOGRAFIA}

1. Beaton GH, Kelly A, Kevany J, Martorell R, Mason J (1990). Appropriate uses of indices in children. ACC/SCN nutrition policy discussion paper $N^{\circ} 7$. Geneva: United Nations.

2. Bénéfice E (1992). Physical activity and anthropometric and functional characteristics of mild malnourished Senegalese children. Ann. Trop. Pediatr. 12: 55-66.

3. Bogin B (1988). Rural-to-urban migration. In C.C.G. Mascie-Taylor and GW Lasker (eds) Biological aspects of human migration. Cambridge: Cambridge University Press, 90-129.

4. Cameron N, Kgamphe JS, Leschner KF e Farrant PJ (1992). Urban rural differences in the growth of South African black children. Ann. Hum. Biol. 19:23-33.

5. Cameron N (1991). Human growth, nutrition and health status in sub-saharan Africa. Yrbk. Phys. Anthropol. 34:21150 .

6. Cameron $\mathrm{N}$ (1992). The monitoring of growth and nutritional status in South Africa. Am. J. Biol. 4: 223-234.

7. Cameron N (1997). Growth and health in a developing country: the South African Experience 1984-1994. In D. F. Roberts, P. Rudan and T. Skaric Croatian Anthropological Society, 131-56.

8. Chaning-Pearce SM, Solomon L (1986). A longitudinal study of the growth of black and white Johannesburg school children. South African Medical Journal 70: 743-746.

9. Corlett JT (1986). Growth of urban school children in Botswana. Ann. Hum. Biol. 13: 73-82.

10. Cortinovis I, Vella V, Ndiku N, Miliani S (1997). Weight, height and arm circumference of children under 5 in the district of Mbarara, south-west Uganda. Ann. Hum. Biol. 6: 557-568.

11. Davies CTM, Mbelwa D, and Dores C (1974). Physical growth and development of urban and rural East African, aged 7-16. Ann. Hum. Biol. 2: 3-12.

12. Davies DP (1988). The importance of genetic influences on growth in early childhood with particular reference to children of Asiatic origin. In J. C. Waterlow (ed.) Linear Growth Retardation in Less Developed Countries. Nestlé Nutrition Workshop Series. Vol. 14: 75-90. New York: Raven Press.

13. Eveleth PB, Tanner JM (1991) Worldwide Variation in Human Growth. 2nd ed. Cambridge: Cambridge University Press.

14. Gillett RM, Tobias PV (2002). Human growth in Southern Zambia: A first study of Tonga children predating the Kariba Dam (1957-1958). Am J Hum Biol 14, 50-60.

15. Goldstein H, Tanner JM (1980). Ecological considerations in the creation and the use of child growth standards. Lancet 15, 582-585.

16. Graitcer PL, Gentry EM (1981). Measuring children: One reference for all. The Lancet 2: 297-299.

17. Habicht JP, Martorell R, Yarbrough C, Malina RM and Klein RE (1974). Height and weight standards of preschool children. The Lancet 6: 611-614.

18. Henneberg M and Louw GJ (1998). Cross-sectional survey growth of urban and rural "Cape Coloured" Schoolchildren: Anthropometry and functional tests. Am. J. Hum. Biol. 10: 73-85.

19. Huttly SRA, Victora CG, Barros FC, Teixeira AM and Vaughan JP (1991). The timing of nutritional status determination: implications for interventions and growth monitoring. Eur. J. Clin. Nutr. 45: 85-95.
20. Keller W (1988). The epidemiology of stunting. In J. C. Waterlow (ed.) Linear growth retardation in less developed countries. Nestlé Nutrition Workshop Series, Vol. 14: 1738. New York: Raven Press.

21. Kow F, Geissler C, and Blasubramaniam E (1991). Are international anthropometric standards appropriate for devoloping countries? J Trop Pediatr 37: 37-44.

22. Lajarraga H (2002). Growth in infancy and childhood: A pediatric approach. In N. Cameron (ed.) Human Growth and Development. New York: Academic Press, 21-44.

23. Malina R (1983). Socio-cultural influences of physical activity and performance. Bulletin de la Societé Belge d'Anthropologie et de Préhistorie 94:155-76.

24. Martins DM (1968). Dynamics of child growth and development in Mozambique. Thesis presented for PhD. at University of Coimbra, Portugal.

25. Martorell R, Fernando M, Ricardo C (1988). Poverty and stature in children. In: JC Waterlow (ed.) Linear Growth Retardation in Less Developed Countries. Nestlé Nutrition Workshop Séries, Vol. 14: 57-71. New York: Raven Press.

26. Martorell R, Khan LK., Schroeder DG (1994). Reversibility of stunting epidemiological findings in children from developing countries. Euro J. Clin. Nutr. 48 (Supp. 1): S45-S57.

27. Meredith HV (1979). Comparative findings on body size of children and youths living at urban centers and in rural areas. Growth 43: 95-104.

28. Monyeki KD, Camelo N, and Getz B (2000). Growth and nutritional status of rural South African children 3-10 years old: The Ellisras growth study. Am. J. Hum. Biol. 12: 42-49.

29. Muria AJ (1998). Efeito das condições sócio-económicas, maturação e do crescimento somático na aptidão física de crianças e jovens da cidade de Maputo de ambos os sexos com idades compreendidas entre os 8 aos 11 anos. Dissertação de Mestrado. Faculdade de Ciências do Desporto e de Educação Física, Universidade do Porto, Portugal.

30. Nkiama E (1993). Croissance, maturation osseuse et performance physique des enfants scolarisés zairois de Bumia agés de 6 a 20 ans. Tese de doutoramento, Universidade Católica de Lovaina, Bélgica.

31. Pawloski LR (2002). Growth and development of adolescent girls from the Segou region of Mali (West Africa). Am. J. Phys. Antr. 117: 364-372.

32. Pawloski LR (2003). Mixed-longitudinal analysis of growth data from Malian adolescent girls: Evidence for compensatory gain? Am. J. Hum. Biol. 15: 178-186.

33. Prista A (1994). Influência da actividade física e dos factores sócio-económicos sobre as componentes da estrutura do valor físico relacionado com a saúde. Estudo em crianças e jovens moçambicanas. Dissertação de Doutoramento. Faculdade de Ciências do Desporto e de Educação Física, Universidade do Porto, Portugal.

34. Prista A (1995). Crescimento, actividade física e aptidão física em países não industrializados: abordagem biocultural em crianças e jovens de Moçambique. Revista Ágon 2: 85-101 (Universidade de Coimbra).

35. Prista A (1998). Nutritional status, physical fitness and physical activity in children and youth in Maputo (Mozambique). In Parizkova J and Hills AP (eds.) Physical fitness and nutrition during Growth. Medicine and Sport Science Reviews, 33: 94-104. Basel: Karger. 
36. Prista A, Marques AT and Maia JAR (1997). Relationship between physical activity, socioeconomic status and physical fitness of 8-15 year old youth from Mozambique. American Journal of Human Biology 9(4):449-457.

37. Prista A, Maia JAR, Marques AT (1998). Sexual dimorphism in physical fitness. A multivariate study. Medicine and Science in Sports and Exercise 5:S155.

38. Prista A, Maia J, Saranga S, Marques A, (2002). Saúde, crescimento e desenvolvimento: Um estudo epidemiológico em crianças e jovens de Moçambique. Porto e Maputo: Faculdade de Ciências do Desporto e de Educação Física, Universidade do Porto; Faculdade de Ciências de Educação Física e Desporto, Universidade Pedagógica de Moçambique.

39. Prista A, Maia JA, Damasceno A, Beunen G (2003). Anthropometric indicators of nutritional status implications for fitness, activity, and health in school-age children and adolescents from Maputo, Mozambique. American Journal of Clinical Nutrition 77 (4): 952-9.

40. Roberts DF (1985). Genetic and nutritional adaptation. In K. Blaxter and J. C. Waterlow (eds.) Nutritional Adaptation in Man. London: John Libby, 45-60.

41. Saranga (2001). Variação de curta duração no crescimento somático e aptidão física de crianças e jovens da cidade de Maputo, Moçambique. Influência das alterações sócio-económicas. Dissertação de Mestrado. Faculdade de Ciências do Desporto e de Educação Física, Universidade do Porto, Portugal.

42. Sellen DW (1999). Growth patterns among seminomadic pastoralists (Datoga) of Tanzânia. Am. J. Phys Anthropol. 109: 187-209.

43. Spurr GB (1988). Body size, physical work capacity and productivity in hard work: Is bigger better? In J.C. Waterlow (ed) Linear Growth Retardation in Less Developed Countries. Nestle Nutrition Workshop Series, Vol. 114: 215-24. New York: Raven Press.

44. Tanner J (1986). Normal growth and technique of growth assessment. Clin Endoc Metab 15(3): 411-451.

45. UNICEF (1994). The state of the Worlds Children 1994. Oxford: Oxford University Press.

46. van Loon H, Saverys V, Vuylsteke JP, and Eeckels R, (1986). Local versus universal growth standards: The effect of using NCHS as universal reference. Ann. Hum. Biol 13: 347-357.

47. Victora CG (1992). The association between wasting and stunding: In international perspective. J. Nutr. 122: 11051110 .

48. Wagstaff L, Reinach SG, Richardson BD, Mkhasibe C, and De Vries G (1987). Anthropometrically determined nutritional status and the school performance of black urban primary schoolchildren. Hum. Nutr. Clinic. Nutr. 41C: 277286.

49. Waterlow JC, Buzina R, Keller W, Lane JM, Nichaman MZ, and Tanner JM (1977). The presentation and use of height and weight data for comparing the nutritional status of groups of children under the age of 10 years. Bulletin of Worth Health Organization 54: 489-498.

50. Waterlow J (1984). Current issues in nutritional assessement by antropometry. In J Borzec and B Schurch (eds.) Malnutrition Behavior: Critical assessemnt and key issues. Lausanne: Nestlé Foundation.
51. World Health Organization (1983). Measuring change in nutritional status. Guidelines for the assessing the nutritional impact of supplementary feeding programmes for vulnerable groups. Geneve: WHO.

52. World Health Organization (1995). Physical status: The use and interpretation of anthropometry. Report of a WHO Expert Committee. WHO Technical Report Séries, 854. Geneve: World Health Organization.

53. WHO Working Group (1986). Use and interpretation of anthropometric indicators of nutritional status. Bulletin of the World Health Organization 64: 929-941.

54. Zverev Y, Gondwe M (2001). Growth of urban school children in Malawi. Ann Hu Biol 4(28): 384-394. 